\title{
Empfehlungen zur Planung und Durchführung des körperlichen Trainings im Lungensport ${ }^{1}$
}

\section{Recommendations for Planning and Realisation of Exercise Training in Outpatient Lung Sports Groups}

Autoren

Institute
O. Göhl' ${ }^{1}$, K. Pleyer ${ }^{2}$, G. Biberger ${ }^{3}$, K. Taube ${ }^{4}$, C. Müller ${ }^{5}$, H. Worth ${ }^{1}$

\author{
Medizinische Klinik I, Klinikum Fürth \\ Hochgebirgsklinik Davos \\ Asthmazentrum Buchenhöhe \\ Atem-Reha Hamburg \\ Fachklinik Allgäu
}

eingereicht 12.6. 2006

akzeptiert 28. 8. 2006

Bibliografie

DOI $10.1055 / \mathrm{s}-2006-944318$

Pneumologie 2006; 60; 716-723

(c) Georg Thieme Verlag KG

Stuttgart · New York

ISSN 0934-8387

Korrespondenzadresse

Dr. phil. Oliver Göhl

Sportwissenschaftler,

Sporttherapeut

Klinikum Fürth, Med. I

Jakob-Henle-Str. 1

90533 Fürth

oliver.goehl@klinikum-

fuerth.de

\section{Zusammenfassung}

Asthma und COPD zählen zu den Volkskrankheiten, die hohe Kosten verursachen. In den nationalen und internationalen Leitlinien wird das körperliche Training als wichtigster nichtmedikamentöser Baustein angeführt. Sowohl bei Asthma bronchiale als auch bei COPD ist evidenzbasiert, dass Effekte in den Bereichen Leistungsfähigkeit, Lebensqualität und gesundheitsökonomische Kosten erreicht werden. Daher sollten flächendeckend Möglichkeiten zur Durchführung des körperlichen Trainings etabliert werden. Das bedeutet, dass neben den stationären Rehabilitationseinrichtungen im ambulanten Bereich vermehrt Lungensportgruppen gegründet werden müssen, die ein kontinuierliches Training ermöglichen. Für diese spezielle Form des längerfristigen Trainings in Gruppen gibt es bisher noch keine systematische Darstellung. Erschwerend sind ferner die unterschiedliche Grundausbildung der Fachübungsleiter und die Heterogenität der Teilnehmer. Absicht der Autoren war es, Empfehlungen für ein gegliedertes und zielgruppenspezifisches Training zu erstellen, um die praktische Umsetzung dem evidenzbasierten Wissensstand anzunähern und zur Qualität des Trainings bei Patienten mit Lungenkrankheiten beizutragen.

\section{Einleitung}

Chronisch obstruktive Atemwegserkrankungen gehören zu den Volkserkrankungen. In Deutschland sind - mit steigender Prävalenz - ca. 10\% der Kinder und 6\% der Erwachsenen an einem Asthma bronchiale erkrankt [1]. In der erwachsenen Bevölkerung sind über 10\% von der COPD betroffen [2]. Beide Erkrankungen verursachen hohe Krankheitskosten, beim Asthma bronchiale ca. 2,1 Mrd. € im Jahr 2002 [1] und bei der COPD schätzungsweise bis zu 8,4 Milliarden $€$ pro Jahr

\section{Abstract}

Asthma and COPD are common diseases inducing high costs. In national and international guidelines exercise training is stated as the main component amongst the nonmedical treatment options. With exercise training beneficial effects in exercise performance, quality of life and healthrelated costs can be obtained. Options for exercise training all over the country should be established. Therefore not only inpatient, but also outpatient structures, especially lung sports groups have to be organized to enable a continuous training. For this special way of long term training no systematic guidelines do exist. Further aggravating factors are the different basic vocational education of the trainers and the heterogeneity of the participants. The intention of the authors was to develop recommendations for a structured training and training sensitive to the target group. The realisation of training with this approach should approximate training to evidence based knowledge and contribute to the quality of training patients with lung diseases. entsprechend $3027 €$ pro Patient und Jahr [2]. Aufgrund der Bedeutung dieser Erkrankungen [3] wurden sowohl auf internationaler als auch auf nationaler Ebene Leitlinien für die Diagnostik und Therapie des Asthmas $[4,5]$ und der COPD $[6,7]$ erarbeitet, welche übereinstimmend die zentrale Bedeutung des körperlichen Trainings im Behandlungskonzept dieser Erkrankungen betonen. Durch Training können sowohl bei

\footnotetext{
${ }^{1}$ Durchführende Institution: Arbeitsgemeinschaft Lungensport in Deutschland e.V.
} 
Asthma bronchiale [8] als auch bei COPD [9] (Evidenzgrad A) die körperliche Leistungsfähigkeit und die Lebensqualität gesteigert sowie Krankenhausbehandlungstage reduziert werden $[10,11]$.

Daher muss körperliches Training ein fester Bestandteil eines multimodalen stationären oder ambulanten Rehabilitationsprogramms sein. Damit diese Effekte langfristig gesichert werden können, ist die Fortsetzung eines körperlichen Trainings in der Rehabilitationsnachsorge und in der ambulanten Versorgung generell flächendeckend und kompetent anzubieten. Eine mittlerweile etablierte und in ihrer Effektivität belegte Methode ist das Training in Lungensportgruppen [12]. Der Vorteil von Lungensportgruppen liegt dabei - auch aufgrund der Förderung gruppendynamischer Prozesse - in der langfristigen und regelmäßigen Durchführung eines effektiven Trainings. Dies ist insofern von Bedeutung, als im Verlauf der Erkrankung nicht nur belastungslimitierende Symptome den Patienten in Vermeidungsstrategien und schließlich in eine Dekonditionierungsspirale führen, sondern auch mit Zunahme von Schweregrad und Symptomatik soziale Isolation, Angstzustände und Depression [13] auftreten.

\section{Aktuelle Situation und Anwendungsbereiche}

In Anlehnung an die Rahmenvereinbarungen zum Rehabilitationssport [14] kann man unter Lungensport Rehabilitationssport für Lungenkranke verstehen. Rehabilitationssport dient dem Zweck, durch den Einsatz von Mitteln des Sports und sportlich ausgerichteter Spiele, die Art und Schwere der speziellen Erkrankung berücksichtigen, eine Verbesserung herbeizuführen. Obwohl noch nicht systematisch untersucht, ist davon auszugehen, dass das Training in den bestehenden ambulanten Lungensportgruppen nicht immer trainingsmethodisch zielgerichtet durchgeführt wird. Erschwerende Faktoren sind die inhomogene $\mathrm{Zu}$ sammensetzung der Gruppen, die geringe Trainingshäufigkeit und die bei diesen Erkrankungen häufigen Exazerbationen und Befindlichkeitsänderungen.

Um in Anbetracht dieser Schwierigkeiten ein möglichst effektives Training sicherzustellen, soll im Folgenden versucht werden, einen Überblick für eine nach trainingsmethodischen Aspekten ausgerichtete Arbeit in den Lungensportgruppen zu geben [15].

\section{Teilnahmevoraussetzungen}

Rehabilitationssport für chronisch kranke Menschen ist in $§ 44$ Abs. 1 Nr. 3 SGB IX festgeschrieben. Vertragliche Grundlage ist die „Rahmenvereinbarung über den Rehabilitationssport und das Funktionstraining vom 1. Oktober 2003“, welche in geänderter Fassung zum 1. Januar 2007 wieder in Kraft treten wird, zwischen den Rehabilitationsträgern (der gesetzlichen Kranken-, Renten- und Unfallversicherung) und den Spitzenverbänden des Sports [14]. Rehabilitationssportgruppen bedürfen in der Regel der Anerkennung durch die zuständigen Behindertensportverbände, wobei die in den Rahmenrichtlinien definierten Voraussetzungen erfüllt sein müssen, damit eine finanzielle Unterstützung durch Kostenträger erfolgt. Der „Antrag auf Kostenübernahme für Rehabilitationssport“ (Formular 56) kann von jedem Arzt ausgefüllt werden, ist nicht budgetpflichtig, aber abrechenbar.
Die medizinischen Voraussetzungen sind ausführlich in den Empfehlungen der Deutschen Atemwegsliga zum Sport und körperlichen Training bei Patienten mit obstruktiven Atemwegserkrankungen [16] dargestellt. Patienten, welche den Aus- und Einschlusskriterien nicht entsprechen, z.B. wegen Vorliegen eines höheren Schweregrades oder spezieller individueller Probleme, sind auf Gruppenfähigkeit zu prüfen und gegebenenfalls Einzeltherapiemaßnahmen oder Rehabilitationsprogrammen zuzuführen. Angesichts der Entwicklung der COPD und der Effekte des körperlichen Trainings wäre für die Zukunft zu überlegen, Lungensport auch für schwergradige Patienten - ähnlich den Übungsgruppen im Herzsport - zu etablieren. Notwendig hierfür sind Vereinbarungen mit Definition z.B. der Rahmenbedingungen.

Entscheidend für die praktische Durchführung sind Art und Ausmaß der funktionellen Beeinträchtigungen sowie vorhandene Komorbiditäten, die in einem Befundbogen „Teilnahmevoraussetzungen für den Lungensport“ erfasst werden. Alle Formulare und weitere Informationen finden sich unter http://www.Lungensport.org.

\section{Krankheitsspezifische Leistungsdiagnostik und Monitoring}

Zielgerichtetes Training setzt Kontrolluntersuchungen voraus. In der pneumologischen Rehabilitation werden unter anderem der 6-Minuten-Gehtest [17], eine Ergometrie oder eine Spiroergometrie [18] als Untersuchungsverfahren zur Angabe der momentanen Leistungsfähigkeit angewandt. Diese leistungsüberprüfenden Belastungstests sollten in regelmäßigen Zeitabständen wiederholt werden.

Monitoringparameter im Rahmen der Trainingseinheiten im Lungensport sollten möglichst einfach zu erfassen sein. Die Beobachtung bzw. Messung verschiedener Körperfunktionen dient der Sicherheit beim körperlichen Training. Bewährt haben sich Herzfrequenz, Laktat, Atemfrequenz, Atemrhythmus, Sauerstoffsättigung, Peakflow-Messung und die Erfassung der Atemnot mit der modifizierten Borg-Skala $[19,20]$. Grenzwerte bei der Belastung sind eine Herzfrequenz von 220 - Alter, eine Sauerstoffsättigung $\leq 90 \%$, ein Abfall der Peak-Flow-Werte um mehr als $20 \%$ des persönlichen Bestwertes und subjektive Atemnot.

\section{Training: Terminologie und Begriffsbestimmung}

Lungensport beinhaltet Training und Übung. Unter Übung versteht man die systematische Wiederholung gezielter Bewegungsabläufe zum Zwecke der Leistungssteigerung durch verbesserte Koordination [21]. Training ist ein komplexer, planmäßiger und sachorientierter Prozess, der eine Verbesserung im jeweiligen Zielbereich anstrebt [22]. Hierbei bedeutet komplex die Auswirkung auf alle leistungsrelevanten Merkmale wie z.B. Funktion der Skelett- und Atemmuskulatur, Beweglichkeit, Ernährungsstatus, Lungenfunktion etc., planmäßig das Einfließen von trainingswissenschaftlichen und krankheitsspezifischen Erkenntnissen z.B. aus der aktuellen Literatur und sachorientiert auf die angestrebte Zielsetzung hinführend wie z.B. physiologischer Trainingseffekt oder Erhalt der Selbstständigkeit. Um konkrete Trainingsempfehlungen geben zu können, ist eine Systematisierung mit einheitlicher Terminologie notwendig ( Tab. 1). 
Tab. 1 Systematisierung und begriffliche Aufarbeitung verschiedener Inhalte körperlichen Trainings im Lungensport

\begin{tabular}{|l|l|}
\hline Bereich & Inhalte (Auswahl) \\
\hline Kraft & $\begin{array}{l}\text { Allgemein: Training mit Hanteln, Theraband, an Krafttrainingsgeräten, etc. Training ohne Gerät in verschiedenen Modalitäten } \\
\text { Speziell: Kraftübungen orientiert an den Aktivitäten des täglichen Lebens }\end{array}$ \\
\hline Ausdauer & $\begin{array}{l}\text { Allgemein: Gehen mit und ohne Stöcken, Ergometertraining, Radfahren, Treppensteigen (cave: Aspekt der Kraftausdauer), } \\
\text { Schwimmen, Bewegungsformen im Wasser, Tanz } \\
\text { Speziell: ADL-orientiertes Ausdauertraining, Rollatortraining }\end{array}$ \\
\hline Beweglichkeit & $\begin{array}{l}\text { Allgemein: Dehnübungen, Übungen zur Mobilisation } \\
\text { Speziell: Dreh- und Dehnlagen, Brustkorbmobilisation }\end{array}$ \\
\hline Koordination & $\begin{array}{l}\text { Allgemein: Übungen zur Schulung der koordinativen Fähigkeiten (Gleichgewichts-, Rhythmisierungs-, Orientierungs-, Um- } \\
\text { stellungs-, Differenzierungs-, Kopplungs- und Reaktionsfähigkeit) mit und ohne Zusatzgeräte. Spiele, Spiel- und Übungsfor- } \\
\text { men mit koordinativen Aspekten, Elemente der Rückenschule } \\
\text { Speziell: Aktivitäten des täglichen Lebens, Atemtechniken bei verschiedenen Belastungsformen }\end{array}$ \\
\hline krankheitsspezifi- & $\begin{array}{l}\text { Selbsthilfetechniken bei Atemnot wie atemerleichternde Körperstellungen und verschiedene Atemtechniken (Ein- und Aus- } \\
\text { atemtechniken wie z. B. Lippenbremse, Stenoseatmung), Hustentechniken, Körperwahrnehmung (speziell Atemwahrneh- } \\
\text { mung), Benutzen verschiedener Devices }\end{array}$ \\
\hline Theorie & $\begin{array}{l}\text { Allgemein: Erläutern von Bewegungsabläufen/Techniken (z. B. Walking, Übungen an Kraftgeräten, Dehnübungen), Wissens- } \\
\text { vermittlung allgemein etc. } \\
\text { Speziell: } \text { Elemente der Patientenschulung, Belastungsreaktionen des Körpers }\end{array}$ \\
\hline
\end{tabular}

Im Rahmen dieser Zuordnung ist die Unterteilung in allgemeine und spezielle Trainingsinhalte notwendig. Bei Atemwegs- und Lungenerkrankungen findet sich die Besonderheit, dass, im Gegensatz zum üblichen methodischen Vorgehen, die Vermittlung von speziellen, krankheitsspezifischen Techniken und das Training der Koordination am Anfang stehen sollten. Damit wird gewährleistet, dass mit einer möglichst optimalen Technik (z.B. Atemtechnik und Körperwahrnehmung) die allgemeinen Trainingsinhalte (z.B. Training der allgemeinen Ausdauer in Form von Gehtraining) gemeistert werden.

Im Rahmen unserer Ausarbeitung wollen wir den Trainingsprozess einer Periodisierung ( = längerfristige Zeitabschnitte z. B. in Form eines Makrozyklus) und Zyklisierung (mittel- und kurzfristige Zeitabschnitte) zuordnen. Der Makrozyklus dient der Ausgestaltung mittelfristiger, das bedeutet mehrwöchiger Abschnitte des Trainingsprozesses mit dem Ziel der Ausformung definierter Entwicklungs- oder Ausprägungsphasen sportlicher Leistungsmerkmale. Ein Mikrozyklus beinhaltet den Zeitraum von 5-10 Tagen, meist 1 Woche. Hilfreich ist hier der Einsatz von Trainingsplänen. Die Trainingseinheit ist die kleinste Einheit, die konkrete Hinweise zur Ausgestaltung gibt sowie einzelne Belastungsziele, Methoden, Inhalt und Mittel, die zur Realisierung benötigt werden, beschreibt.

\section{Methodisches Vorgehen}

Ansatzpunkt unserer Ausarbeitung ist es, bewährte Trainingsinhalte nach dem Prinzip eines Baukastensystems zu kombinieren. Durch eine flexible zeitliche Gliederung in einzelne Makrozyklen, der Umsetzung in verschiedenen wohnortnahen Gegebenheiten und einem Wechsel von Trainingsinhalten-, -mitteln, -methoden und -umfängen können bestehende Defizite der Patienten systematisch angegangen werden. Methodisch soll der Patient unter Berücksichtigung der Ausgangssituation durch Erlernen und Üben einzelner Bausteine mit entsprechendem theoretischen Hintergrundwissen über Körperwahrnehmung zu einem Training einzelner Hauptbeanspruchungsformen (Kraft, Ausdauer, Beweglichkeit, Koordination) und final der Anwendung bei den körperlichen Aktivitäten im Alltag ( $A D L=$ activities of daily living wie z.B. Treppensteigen, Schuhe binden, Anzie- hen) herangeführt werden. Im Rahmen des dargestellten Konzepts ist vorgesehen, dass alle Techniken und Übungen in den Trainingseinheiten der Lungensportgruppe durch den Fachübungsleiter vermittelt und in dem jeweiligen Stundenschwerpunkt trainiert werden. Ergänzende Trainingseinheiten können von dieser Gewichtung abweichen und sollten von den Patienten in Eigenverantwortung nach Rücksprache mit dem Fachübungsleiter durchgeführt werden.

\section{Belastungsnormative}

Die Darstellung notwendiger Trainingsumfänge zum Aufbau und Erhalt der Leistungsfähigkeit erfolgt in Orientierung an internationale, zum Teil nicht krankheitsspezifische Empfehlungen und Statements verschiedener Fachgesellschaften [11,23 - 39]. Hierbei erscheint in den meisten Fällen ein Training an bis zu 3 Tagen/Woche in Haupttrainingsphasen angemessen. Häufigeres intensives Training führt, absolut gesehen, nur zu geringen Steigerungen oder gar keinen Steigerungen der Leistungsfähigkeit [37,40-43]. Ferner steigt das Verletzungsrisiko überproportional $[44,45]$, und die Compliance verringert sich. Erreicht werden sollen diese Trainingshäufigkeiten durch Training in der Lungensportgruppe (in der Regel 1-2 pro Woche), ergänzt um eigenverantwortliche - zum Teil vorgegebene, individuell unterschiedliche - Trainingseinheiten z.B. zu Hause, bei einem freiwilligen Lauftreff oder in einer Gesundheits- und Fitnesseinrichtung.

Eine allgemeine Definition einer Mindesttrainingsintensität in den verschiedenen Bereichen ist angesichts der extremen Bandbreite bei den unterschiedlichen Krankheitsbildern und im Krankheitsverlauf nicht sinnvoll. Explizite Angaben sind entsprechend dem individuellen Leistungsstand zu modifizieren. Auch komplexe Aktivitäten des Alltags wie „Einkaufen“, „Gartenarbeit“ oder ähnliches sind - gerade mit zunehmendem Schweregrad - in der Trainingsplanung zu berücksichtigen. Angesichts der häufig multimorbiden und dekonditionierten Patienten gilt es zu beachten, dass auch ein Ausmaß körperlicher Aktivität, welches nominell unterhalb anerkannter Empfehlungen liegt, dafür aber häufiger, über längere Zeit (pro Trainingseinheit) und mit niedrigerer Intensität durchgeführt wird, 
durchaus die Verbesserungen unter anderem der Leistungsfähigkeit und Lebensqualität bewirkt [46-53]. In der Jahrestrainingsplanung sollten Mindesttrainingsumfänge [54] von ca. 200 Stunden/Jahr berücksichtigt werden. In Haupttrainingsphasen sind 4-6 Stunden Training pro Woche progressiv anzustreben, umgesetzt durch zusätzliches Training in Eigenverantwortung. Je nach Intensität und Umfängen in den einzelnen Trainingsblöcken ist im Vergleich zu Gesunden mit längeren Regenerationsphasen zu arbeiten, da der COPD-Patient durch eine verminderte Regenerationsfähigkeit gekennzeichnet ist [55].

\section{Trainingsblöcke}

Die nachfolgenden Trainingsblöcke sind als Hinweise zur Planung des körperlichen Trainings im Jahresverlauf ohne Bindungscharakter zu verstehen. Ziel ist es, in den einzelnen Blöcken einen Schwerpunkt mit definierten Mindesttrainingsumfängen zu setzen. Gewährleistet wird dies über einen Stundenschwerpunkt der spezifischen Inhalte in den einzelnen Übungsstunden (- Tab. 2) und der oben genannten erweiterten Umsetzung dieser Schwerpunkte in Eigenverantwortung des Patienten.
Tab. 2 Aufbau einer Übungseinheit (60-90 Minuten)

\begin{tabular}{ll} 
Einleitungsphase & $\begin{array}{l}\text { „Unterschreiben“ und Überprüfung der } \\
\text { Befindlichkeit (Teilnahmevoraussetzungen) } \\
\text { zu Stundenbeginn }\end{array}$ \\
\hline Vorbereitungsphase & $\begin{array}{l}\text { Aufwärmen: Allgemein und Speziell } \\
\text { Überprüfung der Befindlichkeit }\end{array}$ \\
\hline Hauptphase & $\begin{array}{l}\text { Stundenschwerpunkt: mind. 50\% der } \\
\text { Unterrichtszeit }\end{array}$ \\
\hline Nachbereitungsphase & $\begin{array}{l}\text { Überprüfung der Befindlichkeit } \\
\text { Ausklang }\end{array}$ \\
\hline Überprüfung der Befindlichkeit
\end{tabular}

\section{Grundlagenblock}

Die Inhalte des Grundlagenblocks ( Tab. 3) stellen einen eigenen Makrozyklus dar. Sie werden in der Regeneration, bei jahreszeitlichen Übergängen und besonders bei Neugründung einer Lungensportgruppe eingesetzt. Ziele sind unter anderem: Erlernen und Festigen aller Techniken und Übungen, Aufbau von Wissen als Grundlage für die Umsetzung in die Praxis (Alltag), Optimierung der Körperwahrnehmung, Wiederannähern an körper-

Tab.3 Grundlagenblock

\section{Bereich}

Theorie

krankheitsspezifische Techniken

Koordination

Kraft

Ausdauer

Beweglichkeit

Monitoring

Trainingshäufig-

keit

Trainingsintensität

\section{Inhalte (Auswahl)}

Elemente der Patientenschulung (v. a. Krankheitsmanagement, Notfallmanagement).

Belastungsreaktion des Körpers z. B. „dynamische Überblähung“, „-Pressatmung“, Herzfrequenz- und Atemfrequenzverhalten, Atemrhythmus.

Erläutern der allgemeinen Bewegungsabläufe in den konkreten Übungen und Trainingsinhalten (z. B. Gelenkpositionierung). Sicheres Führen eines Patiententagebuchs: Peak-Flow, Pulskontrolle, Selbstwahrnehmung, aktuelle Befindlichkeit und Symptome mit dem Ziel, den Patienten zu befähigen, seine aktuelle Sporttauglichkeit zu bewerten und in die Trainingsgestaltung einfließen zu lassen.

Umsetzung: v. a. zu Stundenbeginn und in den Pausen bei Übungen (Orientierung an Patientenschulungsprogrammen). Besonderheiten/Maßnahmen der Differenzierung: Einsatz von schriftlichem Material wie Patienteninformationsbroschüren, Schautafeln etc. Regelmäßige Erfolgskontrollen über „Abfragen“ oder Rollenspiele.

Selbsthilfetechniken wie Ein- und Ausatemtechniken (z. B. Lippenbremse, Hustentechniken, atemerleichternde Körperstellungen), Körperwahrnehmung (speziell Atemwahrnehmung), Handhabung verschiedener Devices und atemphysiotherapeutischer Hilfsmittel.

Umsetzung: als eigenständige Stundenbestandteile oder als Pausengestaltung.

Im Rahmen des Aufwärmens Übungen zur Schulung der koordinativen Fähigkeiten mit Zusatzgeräten oder ohne Geräte. Spiele, Spiel- und Übungsformen mit koordinativen Aspekten zum freudbetonten Wiederannähern an Bewegung, Aktivitäten des täglichen Lebens gekoppelt mit Atemtechniken, Elemente der Rückenschule, etc.

Umsetzung: als eigenständige Stundenbestandteile; in der zeitlichen Abfolge des Trainings kommen die Inhalte der Koordination an erster Stelle zunächst ohne Kombinationsaufgaben.

Erlernen elementarer Übungen mit (Hantel, Theraband) und ohne Gerät (Stabilisierung des Körperstamms, Erarbeiten des aufrechten Sitzes etc.). Dann Erarbeiten von entsprechender Atemtechnik unter Belastung. Ggf. Einsatz externer Stenosen. Umsetzung: als eigenständiger Stundenbestandteil: Nach Erlernen der elementaren Übungen und krankheitsspezifischen Techniken als Inhalt zur Vertiefung und Anwendung. Zunächst Erlernen der Übungen zur Stabilisierung des Körperstamms, dann Übungen mit Gerät und Übungen für die Extremitätenmuskulatur. Im Laufe der Zeit Vorbereiten eines Kraftzirkels. Erlernen kontrollierten Bewegens. Umsetzung der erlernten Übungen und krankheitsspezifischen Techniken durch Gehen, Walking, Treppentraining oder Einsatz von Steppern bei unterschiedlicher Intensität und Dauer. Umsetzung: progressive Belastungssteigerung.

Erlernen elementarer Übungen in den Bereichen Mobilisation und Stretching.

Erlernen verschiedener Dreh- und Dehnlagen.

Umsetzung: Die Inhalte der Beweglichkeit zählen neben denen der Koordination zu den zeitlich gesehen ersten, die geübt werden. Pro Übungsstunde 1-2 Stretchingpositionen der Top-Ten [22] durchführen. Pro Übungsstunde 1 Dreh- bzw. 1 Dehnlage. Besonderheiten/Maßnahmen der Differenzierung: Übungen mit entsprechender Wissensvermittlung kombinieren. Gesamtzustand des Patienten berücksichtigen/erheben (orthopädische Einschränkungen, Probleme beim Hinlegen und Aufstehen) und Umgebungsbedingungen (Temperatur, Lautstärke, etc.) beachten.

Borg-Skala: Atemnot und körperliche Belastung, Symptome, Atemfrequenz und Atemrhythmus, Sauerstoffsättigung, Herzfrequenz, Laktat, Peak-Flow

1 Einheit pro Woche in der Lungensportgruppe

Bei Gruppenneugründung: Individuelles Heimtraining in Form von eigenverantwortlichem Üben

Bei bestehenden Gruppen oder Quereinsteigern: Heimtraining je nach Jahreszeit, Motivation und Befindlichkeit und Person Geringe Intensität 
liche Aktivität und Bewegung, Steigerung des Selbstvertrauens, Compliance bzgl. des Trainings, Motivation und Gruppengefühl. Als Voraussetzung zur Durchführung dieses Blockes empfiehlt es sich, dass der Patient ein strukturiertes, evaluiertes und zielgruppenspezifisches Schulungsprogramm durchlaufen hat. Auch der Fachübungsleiter sollte bei einer solchen Schulung hospitieren.

\section{Ausdauerblock}

Die Inhalte des Ausdauerblocks ( Tab.4) stellen einen eigenständigen Makrozyklus mit dem Ziel der Verbesserung, der Wiederherstellung und des Erhalts der Ausdauerleistungsfähigkeit dar. Voraussetzung sind der Grundlagenblock und hier vor allem sicheres Beherrschen des Notfallmanagements, adäquate Körperwahrnehmung (Symptome, Belastungsreaktionen des Körpers wie z.B. „dynamische Überblähung“) und sicheres Anwenden der krankheitsspezifischen Techniken. Der Patient sollte in der Lage sein, eine Belastung von mindestens sechs Minuten zu tolerieren. Je nach individueller Situation des Patienten kann die Dauer- oder Intervallmethode eingesetzt werden [56-63] ( Tab.4). Patienten mit Flusslimitierung in Ruhe, eingeschränkter körperlicher Leistungsfähigkeit, welche keine Dauerbelastung mit höherer Intensität zulässt, und stark zunehmender dynamischer Überblähung während Belastung scheinen besonders von einer intervallartigen Belastung mit kurzen Intervallen zu profitieren [64].

Es gilt zu beachten, dass ein Training mit hochintensiven Belastungen mit höherem kardiovaskulären Risiko [65], höherem orthopädischen Verletzungsrisiko $[66,67]$ und geringerer Beteili-

Tab. 4 Ausdauerblock

\begin{tabular}{|c|c|}
\hline Inhalte & $\begin{array}{l}\text { Gehen, Laufen, Walking mit Stöcken und ohne } \\
\text { Stöcke, Stepper, Treppensteigen, Ergometer }\end{array}$ \\
\hline Umsetzung & $\begin{array}{l}\text { Intervall- und Dauermethode. } \\
\text { Steigern Trainingshäufigkeit vor Trainingsumfang } \\
\text { vor Trainingsintensität. }\end{array}$ \\
\hline Intensität & $\begin{array}{l}\text { Orientierung an Borg-Skala, Atemfrequenz und } \\
\text { Atemrhythmus, wenn vorhanden Herzfrequenz und } \\
\text { Laktat, bei Dauermethode Intensitäten von } 50-70 \% \\
\text { der Peak-Work-Rate }[50,71] \text { nach entsprechender } \\
\text { Vorbereitung }\end{array}$ \\
\hline Häufigkeit & $\begin{array}{l}\text { Zu Beginn } 1 \times / \text { Woche, ab Woche } 2-3 \text { ca. } 2 \text { Tage/ } \\
\text { Woche, ab Woche } 6 \text { ca. } 3 \times / \text { Woche }\end{array}$ \\
\hline Umfang & $\begin{array}{l}\text { Progressive Steigerung: } z \text {. B. Beginn mit der Inter- } \\
\text { vallmethode }(5 \times 2 \text { Minuten, dann } 2 \times 5 \text { Minuten, } \\
\text { dann } 3 \times 10 \text {, dann } 2 \times 15 \text {, dann } 20+10) \text {, dann Über- } \\
\text { gang auf die Dauermethode }(30 \text { Minuten, > } 30 \text { Minu- } \\
\text { ten) }\end{array}$ \\
\hline Dichte & Individuelle Pausen \\
\hline $\begin{array}{l}\text { Besonderhei- } \\
\text { ten und Maß- } \\
\text { nahmen der } \\
\text { Differenzie- } \\
\text { rung }\end{array}$ & $\begin{array}{l}\text { Ab Schweregrad GOLD III und bei schwergradigem } \\
\text { Asthma und/oder Multimorbidität: eher mit niedri- } \\
\text { geren Intensitäten arbeiten und ggf. bei der Inter- } \\
\text { vallmethode bleiben. } \\
\text { Organisatorisch bietet sich der Einsatz eines Geh- } \\
\text { dreiecks [20] an. Individuelle Vorgaben über ver- } \\
\text { schiedene Bezugsgrößen und deren Relation geben: } \\
\text { Atemfrequenz, Atemrhythmus, Borg-Skala, Herzfre- } \\
\text { quenz. }\end{array}$ \\
\hline Monitoring & $\begin{array}{l}\text { Borg-Skala, Symptome, Atemfrequenz und Atem- } \\
\text { rhythmus, Sauerstoffsättigung, Herzfrequenz, Lak- } \\
\text { tat, Peak-Flow, Blutdruck }\end{array}$ \\
\hline
\end{tabular}

gung [66,68-70] einhergeht. Je nach Erfahrung des Fachübungsleiters, Kenntnis des Patienten (Eingangsdiagnostik unter Berücksichtigung der Ko- und Multimorbidität) und Monitoringmöglichkeiten (v.a. Sauerstoffsättigung, Herzfrequenz) ist differenziert vorzugehen [71,72]. Im Rahmen des Ausdauerblocks sollte ein progressiver Aufbau gewählt werden, z.B. durch Umsetzung von zusätzlichen Ausdauereinheiten.

\section{Kraftblock}

Die Inhalte des Kraftblocks ( Tab. 5) stellen einen eigenständigen Makrozyklus mit dem Ziel der Verbesserung, der Wiederherstellung und des Erhalts der Kraftfähigkeiten dar. Voraussetzung sind der Grundlagenblock und hier v. a. sicheres Beherrschen des Notfallmanagements, adäquate Körperwahrnehmung (Symptome, Belastungsreaktionen des Körpers wie z.B. „Pressatmung“) und Selbsthilfetechniken. Bei COPD bestimmt der Atemrhythmus die Bewegungsausführung.

Das oben beschriebene Vorgehen ist sicher und effizient zur Verbesserung von Kraftfähigkeiten [73-75]. Die Krafttrainingseinheiten sollten auf maximal 60 Minuten inklusive Auf- und Abbau konzipiert werden, da länger andauernde Einheiten mit höheren Ausfallraten einhergehen [66]. Als Mindesttrainingsumfang sollte je 1 Satz pro Übung mit 6-15 Wiederholungen gesetzt werden. Mehr Sätze (multiple-set regimens) können größere Effekte bewirken. Bezüglich der inhaltlichen Gestaltung des Krafttrainings liegen keine evidenzbasierten Angaben vor. Je nach Zielsetzung und Leistungsfähigkeit der Patienten empfehlen sich im Training ein Wechsel von oberer und unterer Ext-

Tab. 5 Krafttrainingsblock

\begin{tabular}{|c|c|}
\hline Inhalte & $\begin{array}{l}\text { Übungen mit Hanteln, Theraband, an Krafttrainings- } \\
\text { geräten etc. Übungen ohne Gerät in verschiedenen } \\
\text { Modalitäten }\end{array}$ \\
\hline Umsetzung & Durchführung: Kraftzirkel mit 8-10 Stationen \\
\hline Intensität* & $\begin{array}{l}\text { Abhängig von Muskelstatus und Ziel: } \\
\text { Kraft-Ausdauertraining: } 15-20 \text { Wdh. und mehr } \\
\text { Muskelaufbautraining: } 6-15 \text { maximale Wdh. } \\
\text { Gewicht fortlaufend anpassen }\end{array}$ \\
\hline Häufigkeit & $\begin{array}{l}\text { Zu Beginn } 1 \times / \text { Woche, ab Woche } 2-3 \text { ca. } 2 \times / \text { Wo- } \\
\text { che, ab Woche } 6 \text { ca. } 3 \times / \text { Woche }\end{array}$ \\
\hline Umfang & 1 später ggf. 2 Durchgänge im Zirkel \\
\hline Dichte & Je nach Wiederholungszahl ca. 3 Minuten Pause \\
\hline $\begin{array}{l}\text { Besonderhei- } \\
\text { ten und Maß- } \\
\text { nahmen der } \\
\text { Differenzie- } \\
\text { rung }\end{array}$ & $\begin{array}{l}\text { unterschiedliche Intensitäten (Gewicht, Farbe des } \\
\text { Therabands, Einbeinig/Einhändig vs. mit beiden Bei- } \\
\text { nen/mit beiden Händen), Geschwindigkeit, ROM } \\
\text { (Range of Motion, Bewegungsumfang), Pausendau- } \\
\text { er, Anzahl der Übungen und Sätze, Bewegungsaus- } \\
\text { führung. Pressatmung vermeiden: bei Belastung } \\
\text { ausatmen und bei Entlastung einatmen. Hilfreich ist } \\
\text { der Einsatz von PEP-Systemen. }\end{array}$ \\
\hline Monitoring & $\begin{array}{l}\text { Borg-Skala, Symptome, Atemfrequenz und Atem- } \\
\text { rhythmus, Sauerstoffsättigung, Herzfrequenz, Lak- } \\
\text { tat, Peak-Flow, Blutdruck }\end{array}$ \\
\hline \multicolumn{2}{|c|}{$\begin{array}{l}\text { * Die maximale Wiederholungsanzahl, die vor Ermüdung durchgeführt } \\
\text { wird, verhindert das Beenden einer weiteren Wiederholung und stellt die } \\
\text { Funktion der Gewichtsbelastung dar. Dies wird als „repetition maximum“ } \\
\text { (RM) bezeichnet. Es spiegelt die Intensität der Belastung wieder. Entspre- } \\
\text { chend wird eine Gewichtslast, welche eine Ermüdung nach der 3. Wieder- } \\
\text { holung bewirkt, als ein „three-repetition maximum“ (3-RM) bezeichnet. Bei } \\
\text { gesunden Personen entspricht dies etwa } 85 \% \text { des Gewichtes, welches ein- } \\
\text { mal bewegt werden kann. }\end{array}$} \\
\hline
\end{tabular}


remität und das Einbauen einer oder mehrerer weniger belastenden Stationen. Pausen sollten bewusst eingehalten werden.

Bevor ein intensives Krafttraining der Extremitätenmuskulatur begonnen werden kann, müssen Bauch-, Rücken-, hintere Schultergürtel- und Gesäßmuskulatur zur Stabilisierung des Körperstamms ausreichend aufgebaut werden. Problematisch erscheint die Umsetzung eines intensiven Krafttrainings. Durch das hohe Ausmaß an Freiheitsgraden ist die exakte Bewegungsausführung beim Krafttraining mit Therabändern und Hanteln nicht unproblematisch. Auch die Dosierung der Belastung bereitet Schwierigkeiten.

\section{Beweglichkeitstraining}

Das Training der Beweglichkeit sollte Bestandteil jeder Übungsstunde sein. Die grundlegenden Übungen werden im Grundlagenblock erarbeitet. Nach dem Erwärmen, zur Pausengestaltung und am Ende der Stunde sollte es gezielt eingesetzt werden. Das Training der speziellen Beweglichkeit bietet sich vor allem in jahreszeitlichen Übergängen (z.B. vom Herbst zum Winter) und Phasen geringer Belastbarkeit an. Bei der Durchführung sollte darauf geachtet werden, dass im Bereich spezielle Beweglichkeit einzelne Dreh- und Dehnlagen 2-5 Minuten gehalten werden. Bewährt hat sich die Kombination mit Atemwahrnehmungsübungen und Elementen der Entspannung. Um die Thoraxmobilität zu erhalten, sollte das Training der speziellen Beweglichkeit kontinuierlich, z.B. ergänzend als Heimtraining, durchgeführt werden. Im Gegensatz zum Training der Kraft und Ausdauer ist hier kein kurzfristiger Trainingserfolg zu erwarten.

\section{Koordinationstraining}

Andere Inhalte zum Training der allgemeinen Koordination (Gleichgewichtstraining etc.) sind sehr geeignet zur abwechslungsreichen Gestaltung der Kurseinheiten z.B. in der Phase des Aufwärmens und dienen u.a. der Sturzprophylaxe. Bei fortgeschrittener COPD kommt dem Training der speziellen Koordination im Sinne von Training der Aktivitäten des täglichen Lebens eine besondere Bedeutung zu. Gemeint ist hierbei insbesondere das Erlernen ressourcenschonender Verhaltensweisen [20] in Kombination mit gezieltem Einsatz von Atemtechniken in Alltagssituationen.

\section{Training im Jahresverlauf}

\section{COPD}

In der Übergangsperiode vom Herbst zum Winter, einhergehend mit erhöhter Infektanfälligkeit, sollte der Grundlagenblock zum Einsatz kommen. Bei stabiler kalter Witterung im Winter ist ein moderates Krafttraining sinnvoll. Eine weitere kritische Phase ist der Übergang vom Winter zum Frühling. Auch hier sollten Elemente des Grundlagenblocks Anwendung finden. Hier sind individuelle Ausnahmen zu gewährleisten. Mit zunehmend stabilem Gesundheitsverlauf in den Sommermonaten empfiehlt sich der Einsatz des Ausdauerblocks und/oder des Kraftblocks. Zur Orientierung zeigt $\bigcirc$ Abb. 1 eine mögliche Gestaltung eines Jahrestrainingsplans. Die Gewichtung der Trainingsinhalte kann durch ergänzende Trainingseinheiten umgesetzt werden, z.B. als Heimtraining, in Gesundheits- und Fitnesseinrichtungen oder im Freien.

\section{Asthma}

Gerade beim Asthma beeinflussen äußere Faktoren das Training [76]. Ein erheblicher Anteil der Asthmatiker leidet an Allergien. Beim Training spielen v. a. spezifische Reize, die saisonal auftreten, wie die der Baum-, Gräser-, Kräuterpollen im Frühjahr und die Schimmelpilzsporen in Sommer und Herbst eine relevante Rolle. Auch unspezifische Reize wie z.B. Kälte und Nebel sind zu beachten. Trotz optimaler medikamentöser Therapie ist dann das Asthma bei vielen der Betroffenen instabiler. Dies resultiert auch in verminderter körperlicher Belastbarkeit. In diesen Zeiten ist den Rahmenbedingungen besondere Achtung zu schenken und adäquat zu reagieren: Durchführung des Sports in der Halle, spezielles Aufwärmen, Reduzierung der Trainingsintensität, Veränderung des Stundenschwerpunkts mit Verlagerung zu weniger asthmaauslösenden Belastungsformen. Außerdem ist eine Intensivierung des Monitorings (Peak-Flow-Kontrolle) notwendig. In kritischen Fällen und bei Infekt können nur Elemente aus dem Grundlagenblock unter Betonung von atemtherapeutischen Inhalten durchgeführt werden. Beim erwachsenen Asthmatiker können in Abhängigkeit von der aktuellen Befindlichkeit folgende Trainingsblöcke als Grundlage für eine Jahresplanung herangezogen werden ( Abb. 2).

\section{Besonderheiten bei Kindern mit Asthma}

Neben den bereits erwähnten spezifischen und unspezifischen Reizen, die zu einer verminderten körperlichen Belastbarkeit führen können, ist beim Sport mit asthmakranken Kindern und Jugendlichen darüber hinaus zu beachten, dass jeder auch unter

\begin{tabular}{|c|c|c|c|c|c|}
\hline & Theorie & Kraft & Ausdauer & $\begin{array}{c}\text { Beweglich- } \\
\text { keit }\end{array}$ & $\begin{array}{c}\text { Krankheitsspezifi- } \\
\text { sche Techniken } \\
\text { Koordination }\end{array}$ \\
\hline Ausdauerblock & $5 \%$ & $15 \%$ & $60 \%$ & $10 \%$ & $5 \%$ \\
\hline Kraftblock & $5 \%$ & $60 \%$ & $10 \%$ & $15 \%$ & $10 \%$ \\
\hline Grundlagenblock & $20 \%$ & $10 \%$ & $5 \%$ & $20 \%$ & $45 \%$ \\
\hline
\end{tabular}

Abb. 1 Entwicklungswürfel COPD. 


\begin{tabular}{|c|c|c|c|c|c|}
\hline & $\begin{array}{c}\text { Theorie und } \\
\text { krankheitsspezifi- } \\
\text { sche Techniken }\end{array}$ & Kraft & Ausdauer & $\begin{array}{c}\text { Beweglich- } \\
\text { keit }\end{array}$ & Koordination \\
\hline $\begin{array}{c}\text { Trainingsblock bei } \\
\text { stabilem Asthma }\end{array}$ & $5 \%$ & $35 \%$ & $45 \%$ & $10 \%$ & $5 \%$ \\
\hline $\begin{array}{c}\text { Trainingsblock bei saisonaler } \\
\text { Beeinträchtigung (spezifische/ } \\
\text { unspezifische Reize) }\end{array}$ & $15 \%$ & $15 \%$ & $15 \%$ & $10 \%$ & $45 \%$ \\
\hline $\begin{array}{c}\text { Trainingsblock } \\
\text { Postinfekt }\end{array}$ & $45 \%$ & $5 \%$ & $5 \%$ & $15 \%$ & $30 \%$ \\
\hline
\end{tabular}

Abb. 2 Entwicklungswürfel Asthma.

Anstrengungsasthma leidet. Um problemlos Sport machen zu können, sollte daher bei der Organisation und Durchführung darauf geachtet werden, dass die medikamentöse Therapie auf das Training abgestimmt ist. Im Bedarfsfall kann eine Prämedikation unmittelbar vor dem Sport notwendig sein. Des Weiteren ist ein mindestens zehnminütiges Aufwärmen zur Vermeidung eines belastungsinduzierten Asthmaanfalles notwendig ( $=$ spezielles Aufwärmen). Intervallartiges Aufwärmen, welches folgendes Prinzip beachtet, hat sich dabei bestens bewährt [76]: Wechsel zwischen Gehen und Laufen im Verhältnis 5:1, z. B. 100 Sekunden Gehen und 20 Sekunden so schnell wie möglich Laufen. Das Aufwärmen sollte mit der Gehphase beginnen und enden. Für die Belastungsphase ist beim Sport mit Kindern noch zu beachten, dass gerade ihre koordinative Entwicklung stark eingeschränkt sein kann. Dem Training der Koordination kommt daher eine weit größere Bedeutung als im erwachsenen Bereich zu. Folgende Gewichtung der einzelnen Trainingsinhalte ist bis zur Erreichung der altersentsprechenden koordinativen Leistungsfähigkeit sinnvoll: Jeweils durchschnittlich können für den Bereich Theorie und krankheitsspezifische Techniken 10\%, für Kraft 15\%, für Ausdauer 25\%, für Beweglichkeit 10\% und für Koordination $40 \%$ verwendet werden. Später können die Trainingsblöcke aus dem erwachsenen Bereich übernommen werden.

\section{Schlussfolgerung}

Die Anzahl der Lungensportgruppen hat in Deutschland in den letzten Jahren deutlich zugenommen, mit weiter steigender Tendenz [77]. Gleiches gilt auch für die Anzahl der ausgebildeten Fachübungsleiter. Außerdem hat sich bei den Teilnehmern im Lungensport das Krankheitsspektrum erweitert und der Krankheitsschweregrad erhöht. Im Hinblick auf die Bedeutung des körperlichen Trainings in der Therapie von chronischen Lungenerkrankungen und der Einführung der Disease Management Programme ist diese Entwicklung dringend erforderlich. Allerdings bedeutet diese Situation für alle Beteiligten, Fachübungsleiter und betreuenden Ärzte auch eine Herausforderung für qualitativ hochwertiges Training. Mit diesen Empfehlungen möchte die Arbeitsgemeinschaft Lungensport in Deutschland Fachübungsleitern Hilfen für ihre praktische Arbeit anbieten. Gleichzeitig sollen Impulse und Grundlagen für die Entwicklung von Qualitätsstandards gegeben werden.

\section{Literatur}

1 Fabel H, Konietzko $N$ eds. Weißbuch Lunge 2005. Stuttgart: Thieme, 2005

2 www.copd.versorgungsleitlinie.de

3 Murray C, Lopez A. Global mortality, disability, and the contribution to risk factors: Global Burden of Disease Study. Lancet 1997; 349: 1436 1442

4 Kardos $P$ et al. Leitlinien zur Diagnostik und Therapie von Asthma. Stuttgart: Thieme, 2005

5 www.ginasthma.com. Global Initiative for Asthma, U.O. Zitat.

6 Worth $\mathrm{H}$ et al. Leitlinie der Deutschen Atemwegsliga und der Deutschen Gesellschaft für Pneumologie zur Diagnostik und Therapie von Patienten mit chronisch obstruktiver Bronchitis und Lungenemphysem (COPD). Pneumologie 2002; 56: 704-738

7 GOLD Global Initiative for Chronic Obstructive Pulmonary Disease. Global strategy for the diagnosis, management, and prevention of chronic obstructive pulmonary disease. NHLBL WHO Workshop Report. Executive Summary. National Institue of Health/National Heart, Lung, and Blood Institute, 2005, Zitat

8 Ram F, Robinson S, Black P. Effects of physical training in asthma: a systematic review. Br J Sports Med 2000; 34: 162 - 167

9 Lacasse $Y$ et al. Pulmonary rehabilitation for chronic obstructive pulmonary disease. Cochrane Database Syst Rev, CD003793, 2002

10 Griffiths $T$ et al. Results at 1 year of outpatient multidisciplinary pulmonary rehabilitation: a randomised controlled trial. Lancet 2000; 355 (9201): $362-368$

11 Behnke $M$ et al. Clinical benefits of a combined hospital and home-based exercise programme over 18 months in patients with severe COPD. Monaldi Arch Chest Dis 2003; 59 (1): 44-51

12 Göhl O. Effekte eines ambulanten wohnortnahen Rehabilitationsprogramms für Patienten mit COPD. Berlin: Lehmanns Media - LOB.de, 2003

13 Mikkelsen $R$ et al. Anxiety and depression in patients with chronic obstructive pulmonary disease (COPD). A review. Nord J Psychiatry 2004; 58 (1): $65-70$

14 Rahmenvereinbarung über den Rehabilitationssport und das Funktionstraining vom 1. Oktober 2003. Zitat

15 Göhl O. Grundlagen der Trainingstherapie bei COPD. Atemw-Lungenkrkh 2005; 31: $501-509$

16 Worth $H$, Meyer A, Folgering $H$ et al. Empfehlungen der Deutschen Atemwegsliga zum Sport und körperlichen Training bei Patienten mit obstruktiven Atemwegserkrankungen. Pneumologie 2000; 54: $61-67$

17 ATS statement. Guidelines for the six-minute walk test. Am J Respir Crit Care Med, 2002; 166 (1): 111 - 117

18 Wassermann $\mathrm{K}$ et al. Principles of Exercise Testing and Interpretation (3rd ed.). Lippincott: Williams \& Wilkins, 1999

19 Borg G. Psychophysical bases of perceived exertion. Med Sci Sports Exerc 1982; 14: 377 - 381

20 Müller C, Jacob S. Sporttherapie bei obstruktiven Atemwegserkrankungen. Großhansdorf: Inter-Pneu-Verlag, 2005

21 Hollmann W, Hettinger T. Sportmedizin. Stuttgart, New York: Schattauer, 2000

22 Weineck J. Optimales Training: Leistungsphysiologische Trainingslehre unter besonderer Berücksichtigung des Kinder- und Jugendtrainings. 13 ed. Balingen: Spitta-Verlag, 2003 
23 American College of Sports Medicine. Position Stand on Progression Models in Resistance Training for Healthy Adults. Med Sci Sports Exerc 2002; 34: 364-380

24 American College of Sports Medicine. American College of Sports Medicine Position Stand. The recommended quantity and quality of exercise for developing and maintaining cardiorespiratory and muscular fitness, and flexibility in healthy adults. Med Sci Sports Exerc 1998; 30: 975 - 991

25 Feigenbaum $M$, Pollock $M$. Prescription of resistance training for health and disease. Med Sci Sports Exerc 1999; 31: 38-45

26 American Association of Cardiovascular and Pulmonary Rehabilitation. Guidelines for Cardiac Rehabilitation Programs. 2nd Ed. Champaign, IL: Human Kinetics Publishers, 1995: 27-56

27 American College of Sports Medicine. Position Stand: Exercise and Hypertension. Med Sci Sports Exerc 2004; 36: 533 - 553

28 American College of Sports Medicine. ACSM position stand on osteoporosis and exercise. Med Sci Sports Exerc 1995; 27: i-vii

29 American College of Sports Medicine. Position Stand: Exercise for patients with coronary artery disease. Med Sci Sports Exerc 1994; 26 : $\mathrm{i}-\mathrm{V}$

30 American College of Sports Medicine. Position Stand: Physical activity, physical fitness, and hypertension. Med Sci Sports Exerc 1993; 25: i-x

31 American College of Sports Medicine. Exercise and Physical Activity for Older Adults. Med Sci Sports Exerc 1998; 30: 992 - 1008

32 Pate $R$ et al. Physical activity and public health: a recommendation from the Centers for Disease Control and Prevention and the American College of Sports Medicine. JAMA 1995; 273: $402-407$

33 NIH Consensus Conference. Physical activity and cardiovascular health. JAMA 1996; 276: 241 - 246

34 Fletcher $G$ et al. Statement on exercise: benefits and recommendation for physical activity programs for all Americans. A statement for health professionals by the committee on exercise and cardiac rehabilitation of the council on clinical cardiology, American Heart Association. Circulation 1996; $94: 857-862$

35 U.S. Department of Health and Human Services. Physical Activity and Health: A Report of the Surgeon General. Atlanta: U.S. Department of Health and Human Services, Centers for Disease Control and Prevention; National Center for Chronic Dosease Prevention and Health Promotion, 1996

36 Gettman $L$ et al. Physiologic responses of men to 1, 3 and 5 day per week training programs. Res Q 1976; 47: 638-646

37 Pollock $M$. The quantification of endurance training programs. In: Wilmore J (ed.). Exercise and Sport Sciences Reviews. New York: Academic Press, 1973: 155 - 188

38 Wenger $H$, Bell G. The interactions of intensity, frequency, and duration of exercise training in altering cardiorespiratory fitness. Sports Med 1986; 3: 346 - 356

39 Nici L et al. American Thoracic Society/European Respiratory Society Statement on Pulmonary Rehabilitation. Am J Respir Crit Care Med 2006; 173: 1390 - 1413

40 Hickson $R$, Rosenkoeter $M$. Reduced training frequences and maintenance of increased aerobic power. Med Sci Sports Exerc 1981; 13: 13 16

41 Hickson $R$ et al. Reduced training duration effects on aerobic power, endurance and cardiac growth. J Appl Physiol 1982; 53: 225 - 229

42 Hickson $R$ et al. Reduced training intensities and loss of aerobic power endurance, and cardiac growth. J Appl Physiol 1985; 58: 492 - 499

43 Martin W et al. Cardiovascular adaptions to intense swim training in sedentary middle-aged men and women. Circulation 1987; 75: 323 330

44 Blair S, Kohl H, Goodyear N. Rates and risks for running and exercise injuries: studies in three populations. Res Q Exerc Sports 1987; 58 : $221-228$

45 Pollock $M$ et al. Effects of frequency and duration of training on attrition and incidence of injury. Med Sci Sports 1977; 9: 31 - 36

46 Hernandez $M$ et al. Results of a home-based training program for patients with COPD. Chest 2000; 118 (1): $106-114$

47 Haskell $W$. Physical activity and health: need to define the required stimulus. Am J Cardiol 1985; 55: 4D - 9D

48 Haskell $W$, Montoye $H$, Orenstein D. Physical activity and exercise to achieve health-related physical fitness components. Public Health Rep 1985; 100: 202 - 212
49 Laporte $R$ et al. The spectrum of physical activity, cardiovascular disease and health: an epidemiologic perspective. Am J Epidemiol 1984; 120 : 507 - 517

50 Paffenbarger $R$ et al. Physical activity and all-cause mortality, and longlivety of college alumni. N Engl J Med 1986; 314: 605-613

51 Vogiatzis I et al. Physiological response to moderate exercise workloads in a pulmonary rehabilitation program in patients with varying degrees of airflow obstruction. Chest 1999; 116 (5): 1200-1207

52 Hui K, Hewitt A. A simple pulmonary rehabilitation program improves health outcomes and reduces hospital utilization in patients with COPD. Chest 2003; 124 (1): $94-97$

53 Ries $A$ et al. Effects of pulmonary rehabilitation on physiologic and psychosocial outcomes in patients with chronic obstructive pulmonary disease. Ann Intern Med 1995; 122 (11): 823-832

54 Zintl F, Eisenhut A. Ausdauertraining. Grundlagen - Methoden - Trainingssteuerung. München-Wien-Zürich: BLV Verlagsgesellschaft $\mathrm{mbH}, 2001$

55 Casaburi $R$. Skeletal muscle function in COPD. Chest 2000; 117 (5 Suppl 1): 267S-271S

56 Casaburi $R$ et al. Physiologic benefits of exercise training in rehabilitation of patients with severe chronic obstructive pulmonary disease. Am J Respir Crit Care Med 1997; 155 (5): 1541 - 1551

57 Debusk R et al. Training effects of long versus short bouts of exercise in healthy subjects. Am J Cardiol 1990; 65: 1010-1013

58 Ebisu T. Splitting the distance of endurance training: effects on cardiovascular endurance and blood lipids. Jpn J Physiol Educ 1985; 30: 37 43

59 Jakicic $J$ et al. Prescribing exercise in multiple short bouts versus one continous bout: effect of adherence, cardiorespiratory fitness, and weight loss in overweight women. Int J Obes 1995; 19: 893 - 901

60 Casaburi $R$ et al. Reductions in exercise lactic acidosis and ventilation as a result of exercise training in patients with obstructive lung disease. Am Rev Respir Dis 1991; 143 (1): 9-18

61 Vogiatzis I, Nanas $S$, Roussos $C$. Interval training as an alternative modality to continuous exercise in patients with COPD. Eur Respir J 2002 20 (1): $12-9$

62 Gosselink R, Troosters T, Decramer M. Exercise training in COPD patients: interval versus endurance training. Eur Respir J 1998; 12: $2 \mathrm{~s}$

63 Pleyer K, Weber M, Schmitz M. Wann Dauer- und wann Intervallmethode? Zur Trainierbarkeit von Patienten mit obstruktiven Atemwegserkrankungen. Z f Physiotherapie 2001; 53: 7

64 Vogiatzis I et al. Dynamic hyperinflation and tolerance to inteval exercise in patients with advanced COPD. Eur Respir J 2004; 24: 385 - 390

65 Siskovick $D$ et al. The incidence of primary cardiac arrest during vigorous exercise. N Engl J Med 1984; 311: $874-877$

66 Pollock $M$. Prescribing exercise for fitness and adherence. In: Dishman R (ed.). Exercise Adherence: Its Impact on Public Health Champaign, IL: Human Kinetics Books, 1988: 259 - 277

67 Pollock M, Wilmore J. Exercise in Health and Disease: Evaluation and Prescription for Prevention and Rehabilitation. 2nd Ed. Philadelphia: WB Saunders, 1990: 91 - 160

68 Dishman $R$, Sallis J, Orenstein $D$. The determinants of physical activity and exercise. Public Health Rep 1985; 100: 158 - 180

69 Martin J, Dubbert P. Adherence to exercise. In: Terjung R (ed.). Exercise and Sport Sciences Reviews. New York: MacMillan, 1985: 137-167

70 Salis J et al. Predictors of adoption and maintenance of physical activity in a community sample. Prev Med 1986; 15: 131 - 141

71 Rochester $C$. Exercise training in chronic obstructive pulmonary disease. J Rehabil Res Dev 2003; 40 (5 Suppl 2): 59-80

72 Puhan MA et al. How should COPD patients exercise during respiratory rehabilitation? Comparison of exercise modalities and intensities to treat skeletal muscle dysfunction. Thorax 2005; 60 (5): 367 - 375

73 Storer $T$. Exercise in chronic obstructive pulmonary disease: resistance exercise prescription. Med Sci Sports Exerc 2001; 33: S680-686

74 Wright $P$ et al. Einfluss eines Krafttrainings auf Lungenfunktionsparameter und Größen der Leistungsfähigkeit von COPD-Patienten. Pneumologie 2002; 56: $413-417$

75 O'Shea SD, Taylor NF, Paratz J. Peripheral muscle strength training in COPD: a systematic review. Chest 2004; 126 (3): 903 - 914

76 Lecheler J, Biberger A, Pfannebecker B. Asthma und Sport. Berchtesgaden: INA-Verlag, 2006

77 www.Lungensport.org 